\title{
Algunas observaciones sobre el tratamiento de la Mola Hidatidiforme
}

(Historias clinicas del Instituto de Protección Materno-Infantil. Años de 1949 y 1950 y método sugerido para su servicio por el Profesor RODRIGUEZ APONTE)

\section{Por el doctor EUDORO CASTILLO VEGA \\ Jefe de Clinica de Maternidad}

I'ara el tratamiento de la Mola Hidatidiforme podemos dividir esta entidad en Mola completa y Mola parcial, entendiendo a la primera como la degeneración total de las vellosidades coriales y la segunda cuando la invasión degenerativa interesa solamente una porción $y$ por lo tanto puede contener embrión, unas reces muerto $y$ atrofiado $y$ en otras ocasiones, excepcionales por lo demás, n embrión puede estar vivo y desarrollarse hasta el final del embarazo.

Desde otro punto de vista, pero también en relación con el tratamiento, se divide la Mola en forma maligna y forma benigna, comprendiendo en la prjmera aquélla en que los tumores quísti. (o-luteínicos del ovario, no regresan después del aborto molar y cuya degeneración conduce al corio-epitelioma: es rsta la Mola Hidatidiforme destructiva, lamada así por algunos autores, y que, revisando las estadísticas nacionales, en Colombia muy pocos casos se han pre- sentado. Esta clase de Mola necesitaríd descle un principio el tratamiento radicaal o sea la Histerectomía total.

La Mola embrionada con feto vivo, requiere la conservación cuidadosa del embarazo hasta una época lo más avanzada posible para asegurar la viabilidad fetal; una vez alcanzado el séptimo mes convendría, a nuestro modo de ver, la interrupción de la gestación con el fin de lograr un niño vivo y desalojar de la matriz las vellosidades quísticas degeneradas. Todo el mundo sabe que el célebre anatomista Beclard, fue producto de un embarazo gemelar, cuya madre había arrojado una Mola Hidatidiforme en el quinto mes de su gestacion.

En la Mola Hidatidiforme total, no embrionada, cuya frecuencia entre nosotros ps de $1,3 \times 1.000$. (Instituto $\mathrm{Ma}$ tcrno-Infantil en Bogotá, sobre un total de 14.831 enfermas, hubo 19 Molas). En contraposición con las estadísticas de Madame Boivin, quien señaló a $1 \times$ 


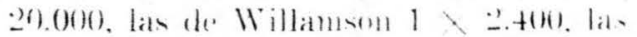

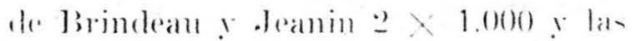

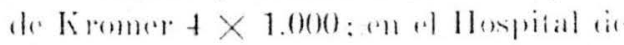

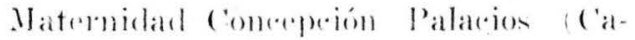

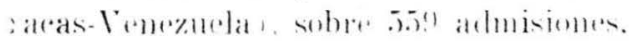

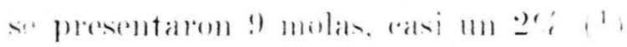

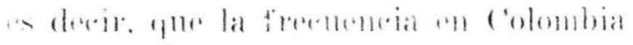
es mayor que las regeistradats ent of exranjero a exoeperom de lemertelat. P'or Io tanto ex para esta clase do Mola en Particular a la que non referimos an este breve eomentario de tratamiento.

In diagnóstico concienzudo de Mola Aidatidiforme indica que el útero debe. s'r evacuado. Esta tesis sostenida per josotros, es la seguida por los clásicos norteamericanos, como por los de la es- vola argentina. Entre nosotros, algu. nos no aronsejan esta medida. sino que wherall que el aborto se realice esponancamente y preconizan tratamiento Indiation y sintomático, mientras tanto.

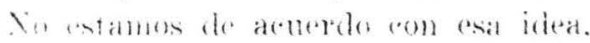

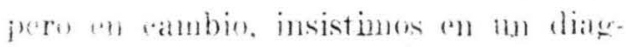
nóstico preseiso: para lo enal no lo danos importancia solamente al exeeso de deserteolle nterino en relación a la elate

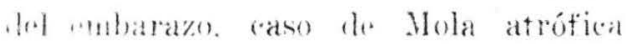

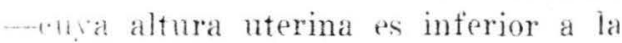
qui enresponde a dieho embarazo-.

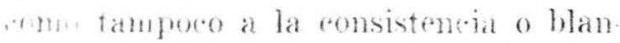
fort wpereal de la matriz y mi signiera a tat hemorratrias de repotietón o la xascratión do los femómenos tóxier. varidioos que deompañan esta gestitción y que se explican por las transfornaviones físico-químicas enalitativas, sine que siempre recurrimos a reforzar huestro eriterio elínico eon la ayuda do laboratorio. Precisa, pute, ma reaceión

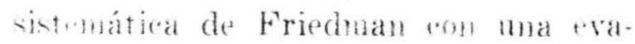

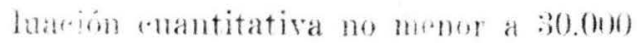

(1) Dato del doctor Oscar Aguero - 1950.

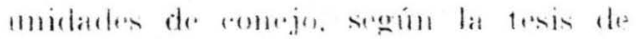
erado del destor . Julio Ménde\% o mat raterein rllantitativa de Galli Mamini

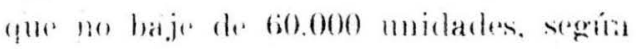
la tesis de grade thel dor.tor . Javier Isazal.

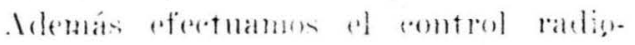
zatien paral sombras fetales, tomamile

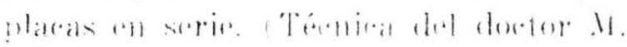
!omillin

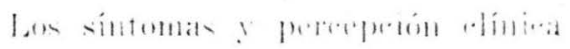
apoyatos por las reateromes biológrase 1 a rontool do radiografia practicados invariablemente a las historias elinieas yue a rontinuarion sintetizo, nos permiieron efortuar sin falla de ninguna lase (2) método Aburel en los casos de Mola Hidatidiforme, así comprobada, con resultados francamente satisfactorios.

En relación a las reacciones biolk, gricas de Ascheim-Zondek, de Friedman. de Hofmamn, de Galli Mainini. se han registrado casos que dieron rosultado negativo en presencia de una Mo. in Hidatidiforme (eitación de De. Jae; ${ }^{1}$ ). Comviene anotar, que para el diagnóstion de la ratidad ine nos ocupa la positividad intensa du las rearejomes biológicas tienen un valor relativo durante los dos primeros meses del embarazo, a causa de la explosión hormonal que tiene lugrar a $\operatorname{los} 60$ días. después del último periodo menstruai; pero, si hallamos valores en las unidades antes anotadas de gonado-tropina, des pués del sexagésimo día, está justificado el diagnóstico de Mola Hidatidiforme.

La evacuación del útero, por los métodos hasta ahora aconsejados como son: e] empleo de laminarias, el uso de bujías dilatadoras, la colocación de globos

(1) Casos registrados igualmente por Josè del C. Acosta y Victor Rodriguez Aponte, en Bogotá. 
6 metremrinteres peopenos. las incisiones de Dürsen o la exarea tagrinal. eran enraminados a vencer mina estre. rher del enello por falia de trabajo o: do iniciación del mismo. si nosotros reitanos las rentraneiones uterinas por medio lel metodo Aburel. al emollo se borra y se reblanderes fisiolegiramente.

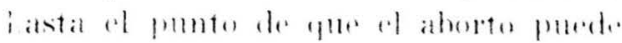

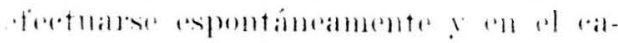

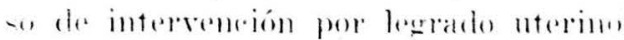
"raspato digital, este se rejenta mureho más fácoll y los resultados obtenidos som ampliamente satisfartorios.

\section{TECNIC.A ABLREL}

Sirguida por nosotios por sugerencia irl Profesor Rodriguez Aponte, original 1. sll aplicacion para Mola Hidatidi. forme.

Plevia confirmación diagnóstica ya nencionada, procedemos a practicar un rateterismo vesical tan completo como sca posible. Aseptizamos la pared abdominal e introducimos una aguja de pun. ión lumbar en el punto medio central de la masa del ovoide uterino previa anestesia local. Aspiramos con jeringa con el objeto de no inyectar en un vaso sanguíneo fetal o uterino: luégo introdueimos de 20 a 100 centímetros, según a) tamaño de la matriz, de una solución hipertónica de cloruro de sodio al 30 o $33 \%$. En ocasiones el útero responde casi immediatamente $y$ en el curso de 24 horas el aborto molar es efectuado. l,uégo ejecutamos una revisión uterina - ch enreta roma o digitalmente como para un aborto incompleto $y$ por precaución dejamos mecha intrauterina impregnada en mertiolato y sulfa en pol10. La mecha es retirada a las 24 horas.
RESIMEN DE IISTORIAS

Historia numero 641.

Nombre: E. T. do 20 años. II gas. talciól.

Entrala, abril $1^{o}$ de 1949 . Salidat. maỵo is le 1!4!?. ('amsat : mejoría.

Reaceión de Friedman, enantitativa. positive para solo(n) l'. eoner.jo.

sulimentarion: :3:- $\mathrm{mm}$. en $1 / 2$ hora. is mom. a la bora ( 1 ).

(madro hemátion: 4.080. mon por smm:. Lenroritos. T.600. Hemoglobina, ×5. Valor grobular, 1,06. Polinueleares uentrófilos, 60\%. Eosinófilos, 4\%. Liniocitos. $36 \%$.

Radiografía: no so observan partes fotales.

I. R.: no recuerda. Vómitos intensos

$\checkmark$ hemorragias de repetición.

Vtero: altura 22. Consistencia pastom:

Diagnóstico Mola Midatidiforme.

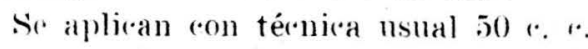
de la solución hipertónica en eloruro dr. sodio al $30 \%$. Las contraceiones se inirian en media hora.

El aborto se verifica a las 16 horas y se eompleta con rasparlo digital y taponamiento.

Historia múmero 1.319 .

Nombre: M. del C. M., de 25 años. 11 gestación.

Entrada, junio 30 de 1949. Salida, julio 23 de 1949. Causa: mejoría.

U. R.: febrero 16 de 1949. Causa de ingreso: Hemorragia escasa de sangre roja, desde hace un mes. Pequeños do-

(1) Es de anotar el valor alto en la Eritrosedimentación, digno de tenerse en cuenta desde el punto de vista de ayuda del Laboratorio a la Clínica. 
'ores sordos e intermitentes en la región l:ipogástrica. Edemas miembros inteyiores.

Altura uterina: 27 centímetros. Consistencia pastosa. No se aprecian partes frtales ni ruidos. T. A. $171 / 2 \times 10$.

Serológico: negativo.

Reaceión de Friedman, euantitativa Jositive para $50.000 \mathrm{l}$. (eont.jo.

Radiografía: no se aprecian partes tetales.

Tratamiento: lo mismo que la historia anterior.

IIistoria número 783.

Nombre: A. L. M., de 28 años. V ges. tación.

Entrada, abril 19 de 194! Salida, maye 6 de 1949. Causa: mejoría.

I. R. : enero $11 / 4$. Causa de ingreso. hemorragia pequeña desde hace un mes.

Altura uterina: 21 centimetro. Consisten.ia: no muy blanda, irregular (i).

Reacción de Friedman: positivo para 50.000 unidades.

Radiografía: no se aprecian partes fetales.

Tratamiento: Aburel (nisma técai $(\cdot a)$

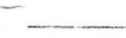

\section{Historia número 751.}

Nombre: M. C.. de 18 años. Il geritación.

Entrada, abril 16 de 1949 . Salida, 22 de abril de 1949. Causa: mejoría.

I. R. : noviembre 16 de 1948. Causa (ie ingreso: estado nauseoso. Inapetenaia. Homorragia con coágulos que repi-

(1) Anótese la consistencia poco blanda de este caso y la irregularidad de sus contornos. e. Llega en commenzo de trabajo. L'or 1) tacto, se hace diagnóstico de iniciat(ión de aborto molar.

Se hace Aburel y el aborto se declara francamente. Se hace taponamiento.

Historia número 689.

Nombre: D. M. S., de 22 años. 1 gess1atción.

Entrada, abril 11 de 1950. Salida, abril 19 de 1950. Causa: defunción.

$\mathrm{U}$. R. : dice que hace 15 días.

Llega de la Clínica Tropical. Enferuna retrasada mental.

Altura uterina: 23 centímetros. No liay rúdos ni se aprecian partes fetales.

Galli Mainini, positivo para 50.000 imidades.

Radiografía: negativo para feto. I $1 \dot{t}$ de abril de 1950 .

Masa abdominal de 23 centímetro de altura. Regular, ligeramente reni nte - forma de corazón de naipe-. Farece que se contrae.

Se hizo el raspadio hasta el 19 de abril. Se comprobó la expulsión de Mola. Pero el tratamiento fue tardío.

Se contemporizó en la creencia de un aborto espontáneo.

Muerte por anemia, según necropsia.

Historia número 237.

Nombre: R. G., de 19 años. I gestación.

Entrada, febrero 6 de 1950. Salida, febrero 13 de 1950. Causa: mejoria.

Causa de ingreso: flujo sanguinolento desde hace un mes $y$ dolores intermitentes a la cadera.

U. R.: agosto 12 de 1949. 'T. A. $14 \times 9$. 
Aluma nterina: 12 entimetros Con. sistencia: blanda.

No se aprecian ni ruídos ni parto tetales.

Reareión de Fredman : positivo para i).000 I: ronejo.

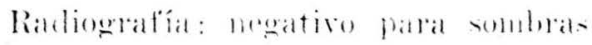
stakles.

Tratamiento: Aburel.

Historia rúmero 1.047 .

Nombre: A. S., de 35 años. IV gestación.

Eutrada. mayo 26 de 1950. Salicla. junio ti de 1950. C'allsa : mejoría.

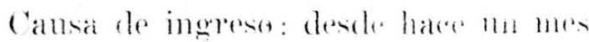

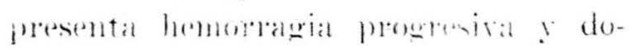
!ores.

U. R. : febrero $1^{\circ}$ de 195 ).

Altura uterina: 18 centímetros - remitente- No hay ruídos fetales. Cuello de multípara blando. Orificio inter1:0 cerrado. Hemorragia raginal. Se ordena progesterona. Mientras llegan resultados de laboratorio.

Galli Mainini: positivo para $80.0(n)$ midades.

Radiografía uterina: no se apretian nombras fetales.

Se have el diagnóstico de Mola y se instala el tratamiento Aburel. Luégo raspado digital y taponamiento.
Historia número 2.641.

Nombre: J. G., de 28 años. VIl gastación.

Entratlat dieciembere 26 de 19.50. Salidia curo 20 de 1951. Causa: mejoría.

(ansia de ingreso: hemorragias de reveticion. Vomites.

I. Ri.: 1.) de ageosto de 1950. No se apretian ruídos ni partes fetales.

Datos rlínicos: positivos para Mlula.

Radiografía: no se aprecian partes fetales.

No se hizo Friedman.

Se instala el tratamiento Aburel. Raslado y taponamiento.

\section{BIBLIOGRAFIA}

1 Confereneias de Clínica Obstétriea Profesor Rodríguez Aponte. (1949 Y 1950 ).

- Revista en la Sociedad de Obstetricia y Ginecología de Venezuela. Doc. tor Oscar Aguero, No 4 de 1950.

3 Estadísticas del Instituto de Protección Materno-Infantil, en Bogotá.

4 La Rearción de Friedman. Tesis de Grado de Julio Méndez Barreneche.

5 La Reacción de Galli Mamini. Tesis d. Grado de Javier Izasa.

$\therefore$ Principios y práctica de Obstetricia 11 tomo. por De Lee-(ireenhill.

7 Tratado de Obstetricia. Il tomo, por Mammel L. Pérez.

$s$ Ohstetricia. II tomo, por Williams. 